\title{
Changes in proinflammatory cytokines and white matter in chronically stressed rats
}

\author{
This article was published in the following Dove Press journal: \\ Neuropsychiatric Disease and Treatment \\ 6 March 2015 \\ Number of times this article has been viewed
}

\section{Ping Yang ${ }^{\prime}$ \\ Zhenyong Gao' \\ Handi Zhang' \\ Zeman Fang' \\ Cairu Wu' \\ Haiyun $\mathrm{Xu}^{1,2}$ \\ Qing-Jun Huang'}

'Mental Health Center, ${ }^{2}$ Department of Anatomy, Shantou University Medical College, Shantou, Guangdong, People's Republic of China
Correspondence: Haiyun Xu; Qing-Jun Huang

Mental Health Center, Shantou University Medical College, 22 Xinling

Road, Shantou, Guangdong 51504I,

People's Republic of China

Tel +86 75488900728

Email hyxu@stu.edu.cn;

huangqj@stumhc.cn

\begin{abstract}
Although the pathogenesis of depression, an incapacitating psychiatric ailment, remains largely unknown, previous human and animal studies have suggested that both proinflammatory cytokines and altered oligodendrocytes play important roles in the condition. This study examined these two factors in the brains of rats following unpredictable chronic mild stress for 4 weeks, with the hypothesis that chronic stress may affect oligodendrocytes and elevate proinflammatory cytokines in the brain. After suffering unpredictable stressors for 4 weeks, the rats showed depression-like behaviors, including decreased locomotion in the open field, increased immobility time in the forced swim test, and decreased sucrose consumption and less sucrose preference when compared with controls. Immunohistochemical staining of brain sections showed higher immunoreactivity of proinflammatory cytokines in certain brain regions of stressed rats compared with controls; lower immunoreactivity of myelin basic protein and fewer mature oligodendrocytes were seen in the prefrontal cortex, but no demyelination was detected. These results are interpreted and discussed in the context of recent findings from human and animal studies.
\end{abstract}

Keywords: cytokines, depression, myelination, oligodendrocytes, stress

\section{Introduction}

Depression is an incapacitating psychiatric ailment characterized by a pervasive low mood, loss of interest in usual activities, anhedonia, withdrawal of interest, feelings of worthlessness, and a suicidal tendency. ${ }^{1,2}$ The pathogenesis of this psychiatric disorder remains largely unknown. Of the proposed players, proinflammatory cytokines are important in mediating behavioral, neuroendocrine, and neurochemical features of depressive disorders. ${ }^{3,4}$ In line with this point of view, patients with depression frequently show alterations in their immune system, such as elevated proinflammatory cytokine levels in plasma and cerebrospinal fluid. ${ }^{5}$ In addition, administration of interferon- $\alpha$ in patients with cancer led to high depression rates, ${ }^{6,7}$ whereas inhibiting proinflammatory cytokines or their signaling pathways improved depressed mood and the response to conventional antidepressant medications..$^{5,8}$ More significantly, some antidepressant drugs reduced the release of proinflammatory cytokines but increased the release of endogenous antagonists of proinflammatory cytokines, such as interleukin (IL)-10. ${ }^{9}$

Another important advance in our understanding of the pathophysiology of depression resulted from the development and application of new magnetic resonance imaging methods such as diffusion tensor imaging and magnetization transfer imaging in psychiatry research over the past two decades. Human studies using these new neuroimaging methods have highlighted white matter pathology in psychiatric disorders including schizophrenia, bipolar disorder, major depressive disorder (MDD), 
and anxiety disorder. In diffusion tensor imaging studies, fractional anisotropy is a quantitative index of white matter coherence and integrity. Lesions in white matter structures generally produce reductions in fractional anisotropy. There is an increasing number of human studies in this area, with reports of MDD patients having lower fractional anisotropy values in the cortical and subcortical brain regions, including the frontal gyrus, ${ }^{10,11}$ parietal lobe and occipitotemporal gyrus, ${ }^{11,12}$ inferior parietal portion of the superior longitudinal fasciculus and anterior limb of the internal capsule, ${ }^{13}$ and left striatum. ${ }^{14}$ In line with these neuroimaging studies, post-mortem studies reported a lower density of oligodendrocytes (OLs) in layer VI of the prefrontal cortex (PFC) in subjects with $\mathrm{MDD}^{12,15}$ and a significant decrease in expression of genes related to OL function in the temporal cortex of MDD patients. ${ }^{16}$

In parallel with human studies, recent animal studies have provided evidence associating proinflammatory cytokines with the pathophysiology of depression. For example, when challenged with a social stressor, mice showed an increase in cytokines and corticosterone levels in peripheral blood. ${ }^{17}$ Moreover, central administration of lipopolysaccharide induced depressive-like behaviors in mice, while the treatment increased steady-state transcripts of tumor necrosis factor (TNF)- $\alpha$, IL-6, and the inducible isoform of nitric oxide synthase in the hippocampus. ${ }^{18}$ Central administration of IL-1 $\beta$ also induced sickness and depressive-like behaviors and elevations of IL-6. ${ }^{19}$

In line with the neuroimaging findings in subjects with MDD as reviewed above, rodents exposed to chronic stress showed less proliferation of OLs and NG2-positive cells in the frontal cortex, and this pattern was reversed by antidepressant treatments. ${ }^{20,21}$ Similarly, chronic corticosterone exposure in rodents decreased cortical and amygdala $\mathrm{OL}^{20}$ and NG2-positive cell proliferation. ${ }^{22,23}$ These findings suggest that OLs may be particularly susceptible to stress-related and corticosterone-induced toxicities and that OLs may be targeted by antidepressant treatments.

The above-mentioned human and animal studies suggest that both proinflammatory cytokines and OLs are two important players in the pathophysiology of depression. However, there is no direct evidence of concurrent white matter abnormality and elevated proinflammatory cytokines in the brains of patients with MDD and animal models of depression. The aim of this study was to examine these factors in the brains of stressed rats. For this purpose, an unpredictable chronic mild stress (UCMS) model was employed (see Materials and methods section). It is a validated rodent model of depression and has been used to elucidate the role of neuroinflammation in the pathophysiology of depression ${ }^{24}$ and to test the effects of antidepressant treatments. ${ }^{25-27}$

\section{Materials and methods}

\section{Animals and experimental design}

Male Sprague-Dawley rats (aged 8 weeks, 220-270 g) were purchased from the Animal Center of Shantou University Medical College. They were housed with a regular light-dark cycle (lights on at $7 \mathrm{am}$, lights off at $7 \mathrm{pm}$ ) at a controlled temperature $\left(22^{\circ} \mathrm{C} \pm 1^{\circ} \mathrm{C}\right)$ and humidity $(50 \% \pm 5 \%)$, and were given a standard diet and water ad libitum. After acclimatization for 7 days, the rats were randomly assigned to either a control or stressed group ( $n=8$ per group). One day after the last stressor, the animals were subjected to behavioral tests, ie, open-field, forced swim, and sucrose consumption, in order. There was an interval of 24 hours between two behavioral tests. These arrangements (order and interval of behavioral tests) were expected to minimize the stressful effects of one behavioral test on the following one. One day after the last behavioral test, the rats were sacrificed under deep anesthesia and their brains were processed for histochemical and immunohistochemical staining to localize and measure the expression of proinflammatory cytokines and evaluate the status of white matter in different brain regions. For this purpose, we used immunohistochemical staining rather than Western blot analysis. All animal procedures used in this study were in accordance with the guideline set up by the Animal Care and Use Committee of Shantou University Medical College and approved by the ethics committee.

\section{UCMS procedure}

All rats in the stressed group were housed individually in small cages $(24 \mathrm{~cm} \times 11 \mathrm{~cm} \times 12 \mathrm{~cm})$ and subjected to various stressors according to a "random" schedule for 4 weeks. The UCMS procedure was as described before ${ }^{25}$ with a slight modification. The following stressors were administered in random order: food deprivation (24 hours), water deprivation (24 hours), social stress (switching cages), cage tilting for 24 hours $\left(45^{\circ}\right)$, overnight illumination, wet bedding for 10 hours, swimming in $4^{\circ} \mathrm{C}$ water for 5 minutes, tail pinch for one minute, and restraining in a $300 \mathrm{~mL}$ tube for 2 hours. The rats were subjected to one of these stressors at a different time each day and the same stressor was not applied consecutively on 2 days so the animals could not predict the occurrence of any stimulation. In addition, food and water 
deprivation did not follow one another consecutively to minimize the nutritional stress on the animals. The control group was undisturbed except for necessary procedures, such as routine cage cleaning.

\section{Open-field test}

This test was carried out to measure exploratory behavior and locomotor activity in the rats. To adapt to the environment, all rats were placed in the test room for one hour before starting the test. Each rat was placed at the center of the open field $(100 \mathrm{~cm} \times 100 \mathrm{~cm} \times 65 \mathrm{~cm})$ and allowed to move freely around it and to explore the environment for 5 minutes. The travel paths of the animals were recorded by a video camera placed above the arena. The video tracking system (DigBehav System, Yishu Co Ltd, Shanghai, People's Republic of China) was used to measure the total distance, distance traveled in the central zone, and retention time in the central zone. The ratio of distance traveled in the central zone over the total ambulation distance was calculated. The arena was cleaned with $70 \%$ alcohol after every test.

\section{Forced swimming test}

This test was performed using a cylindrical glass tank (height $65 \mathrm{~cm}$, width $30 \mathrm{~cm})$ filled with warm water $\left(24^{\circ} \mathrm{C} \pm 1^{\circ} \mathrm{C}\right)$ to a depth of $40 \mathrm{~cm}$. It consisted of a 15-minute pretest swim and a 5-minute test swim 24 hours later. The water was changed for each animal. After the test, the rat was dried in a warm cage, and then returned to its home cage. The floating time (immobility) during the test period was recorded. The rat was considered immobile when it floated in the water and only performed movements enabling it to keep its head above the water.

\section{Sucrose consumption test}

This test was used to determine anhedonia, ${ }^{28}$ one of the core symptoms of MDD in humans. Rats were trained for 48 hours to adapt to $1 \%$ sucrose solution (w/v). During the training period, each individually housed rat was able to access both the sucrose solution and tap water; the location of the two bottles was exchanged once every 12 hours. After adaptation, the rats were deprived of water and food for 21 hours and then allowed to consume the sucrose solution and tap water ad libitum for 3 hours. At the end, sucrose and water consumption $(\mathrm{mL})$ was measured and the sucrose preference was calculated as a percentage of the consumed $1 \%$ sucrose solution relative to the total volume of liquid intake.

\section{Histological and immunohistochemical staining}

One day after the last behavioral test, the rats were deeply anesthetized with $2 \%$ pentobarbital and perfused through the left ventricle with saline, followed by $4 \%$ paraformaldehyde in phosphate buffer. Their brains were then removed and post-fixed overnight in the same fixative, followed by cryoprotection in $20 \%$ and $30 \%$ sucrose at $4{ }^{\circ} \mathrm{C}$ until they sank to the bottom. Serial $25 \mu \mathrm{m}$ coronal sections of the brains were cut using a cryostat microtome (Leica, Wetzler, Germany) and mounted on gelatin-coated slides. One set of sections was used to evaluate demyelination using Luxol fast blue-periodic acid Schiff (LFB-PAS) staining. Other sets of sections were used for immunohistochemical staining.

For LFB-PAS staining, brain sections were hydrated in $95 \%$ alcohol and incubated in $0.1 \%$ LFB solution overnight at $58^{\circ} \mathrm{C}$. The sections were then differentiated in a lithium carbonate solution $(0.05 \%)$ and alcohol $(70 \%)$, followed by counterstaining with PAS.

For immunohistochemical staining, brain sections were pretreated with $0.3 \%$ hydrogen peroxide in methanol for 30 minutes, washed in phosphate-buffered saline, and incubated in $0.3 \%$ Triton $\mathrm{X}-100$ for 10 minutes at $4{ }^{\circ} \mathrm{C}$. After washing with phosphate-buffered saline, sections were incubated for one hour at $22^{\circ} \mathrm{C}$ with a blocking solution composed of $10 \%$ normal goat serum and $5 \%$ bovine serum albumin. Sections were then incubated with a primary antibody to glutathione-S-transferase- $\pi$ (1:100, Boster, Wuhan, People's Republic of China), myelin basic protein (MBP, $1: 100$, Boster), TNF- $\alpha$ (1:100, Boster), IL-1 $\beta$ (1:100, Abcam, Cambridge, MA, USA), or IL-6 (1:100, Abcam) in the blocking solution for 48 hours at $4^{\circ} \mathrm{C}$. After rinsing in phosphate-buffered saline, the sections were incubated in horseradish peroxidase-labeled anti-rabbit immunoglobulin $\mathrm{G}$ (Zsbio, Beijing, People's Republic of China) for 30 minutes at $37^{\circ} \mathrm{C}$. After rinsing in phosphate-buffered saline, the sections were visualized using DAB (Maxvision Technology, Futian, People's Republic of China) as the chromogen. The negative controls were prepared using the same procedure as described above, except for omission of the primary antibodies. No positive immunostaining was found in any of the controls.

\section{Image analysis}

Six coronal brain sections from each animal in the control group and the stressed group were examined. Digital images were recorded using an $\mathrm{H} 550 \mathrm{~L}$ research microscope (Nikon, Sendai, Japan). These sections are between positive $4.70 \mathrm{~mm}$ 
and minus $4.80 \mathrm{~mm}$ from bregma ${ }^{29}$ and $75 \mu \mathrm{m}$ apart. Some of brain areas/structures in this part of the brain, including the PFC, corpus callosum, internal capsule, external capsule, caudate putamen (CPU), hippocampus, and hypothalamus, were examined for immunoreactivity of the cytokines IL-6, IL-1 $\beta$, and TNF- $\alpha$ and for white matter status (immunohistochemistry of MBP and glutathione-S-transferase- $\pi$ and LFB-PAS staining) using Image-Pro Plus version 6.0 software (Media Cybernetics, Silver Spring, MD, USA). The corpus callosum, internal capsule, and external capsule are important white matter structures, which contain no neurons but do contain neuroglial cells, so are suitable for checking white matter status as reported in previous studies. ${ }^{30-32}$ The CPU is a brain region occupied mainly with myelinated fibers that can be readily visualized histochemically and immunohistochemically. The PFC and hippocampus are two brain regions that are most vulnerable to depressive interferences and are affected in patients with MDD. ${ }^{33-35}$ In the hypothalamus, measurement was focused on the ventromedial hypothalamus (VMH). In a previous study, ${ }^{36}$ this subregion of the hypothalamus was shown to respond to chronic stress by increasing the expression of some proinflammatory cytokines.

The same conditions were maintained for measurement of all the selected brain sections and the values were read out automatically by the software. The immunoreactivity of MBP and TNF- $\alpha$ in a stressed rat was normalized as the percentages of the same indices in a control, whereas numbers of IL-1 $\beta$, IL- 6 , and glutathione-S-transferase- $\pi$ positive cells of all animals were counted and expressed as cells $/ \mathrm{mm}^{2}$.

\section{Statistical analysis}

The data were analyzed using Statistical Package for the Social Sciences version 19 (IBM Corporation, Armonk, NY, USA) and expressed as the mean \pm standard error of the mean. The independent-samples $t$-test was used to compare sets of data between the control and stressed rats. Differences were considered statistically significant at $P<0.05$.

\section{Results}

\section{Behavioral changes caused by UCMS}

As shown in Figure 1A, rats in the UCMS group traveled a shorter distance in the whole open field $(t=4.99, P<0.01)$ when compared with controls. The ratio of the central distance over the total distance in the stressed rats was also significantly lower than in controls ( $t=7.93, P<0.001$; Figure 1B). The stressed rats spent less time in the central zone compared with controls ( $t=6.58, P<0.001$; Figure 1C).
A

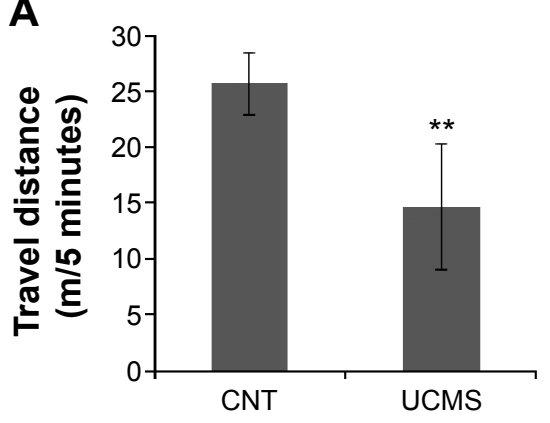

D

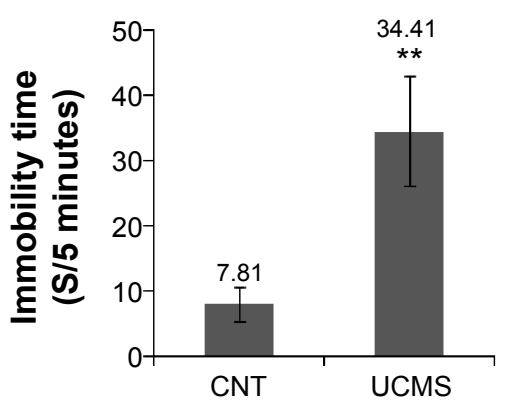

B

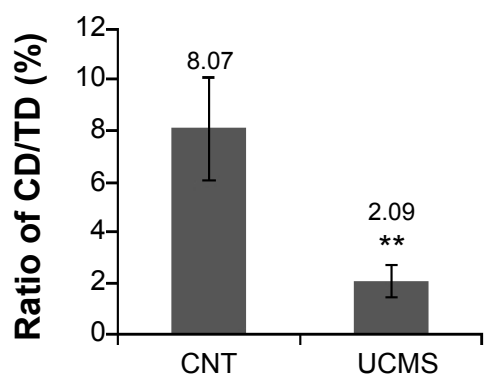

E

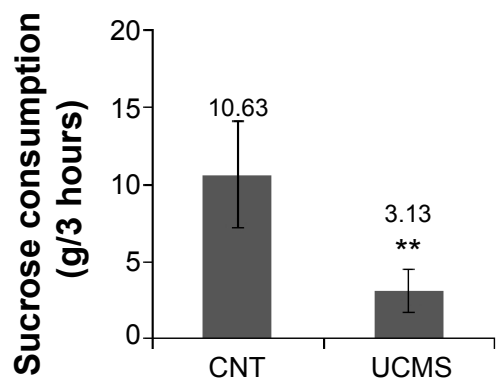

C

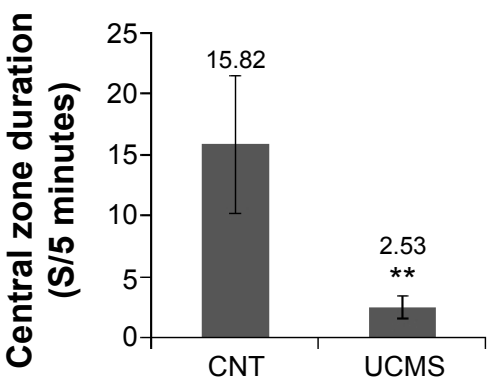

$\mathbf{F}$

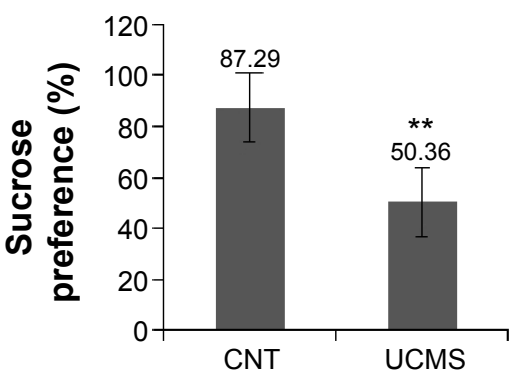

Figure I Depression-like behaviors caused by UCMS.

Notes: After chronic unpredictable stress for 4 weeks, rats showed shorter distances traveled in the central zone and the whole open field (A), a lower ratio of central distance over total distance (B), less time spent in the central zone of the open-field (C), a longer period of immobility in the forced swimming test (D), less consumption of sucrose $(\mathbf{E})$, and a lower preference for sucrose $(\mathbf{F})$ when compared with the CNT group. The data are summarized as the mean $\pm s t a n d a r d$ error of the mean. $* * P<0.01$. Abbreviations: CD/TD, central distance/total distance; CNT, control; UCMS, unpredictable chronic mild stress. 
In the forced swim test, stressed rats showed a longer immobility time compared with the controls $(t=8.54$, $P<0.001$; Figure 1D).

In the sucrose consumption test, stressed rats consumed a smaller amount of sucrose than controls (Figure 1E), with a significantly lower percentage of consumed 1\% sucrose solution over total volume of liquid intake (sucrose preference) than the controls $(t=5.03, P<0.001$; Figure $1 \mathrm{~F})$.

\section{Elevated proinflammatory cytokines caused by UCMS}

Higher numbers of IL-1 $\beta$-positive cells were found in the CPU, hippocampus (including CA1, CA3, and dentate gyrus), and VMH of stressed rats, but no differences were found in the other brain areas between the two groups (Figure 2). Also, many more IL-6-positive cells were found in the CPU and VMH of stressed rats, while the other brain areas were comparable between the two groups (Figure 3 ). Interestingly, TNF- $\alpha$ immunoreactivity was found to be higher in the PFC, hippocampus (including CA1, CA3, and dentate gyrus), and $\mathrm{VMH}$ in the stressed rats, while no differences between the two groups were found in the other brain areas (Figure 4).

\section{Decreased MBP immunoreactivity and OLs caused by UCMS}

LFB-PAS staining showed no demyelination in the examined brain regions of stressed rats (data not shown). However, MBP immunoreactivity was significantly decreased in the PFC and external capsule of stressed rats when compared with controls. In this regard, the other brain regions were comparable between the two groups (Figure 5). As expected, numbers of OLs in the PFC were also significantly decreased in stressed rats compared with controls, but no difference between the two groups was found for this index in the external capsule (Figure 6).

\section{Discussion}

In accordance with previous studies, ${ }^{24-26,37,38}$ UCMS induced depression-like behaviors in rats in the present study, including decreased locomotion in the open field test, increased immobility time in the forced swim test, and decreased

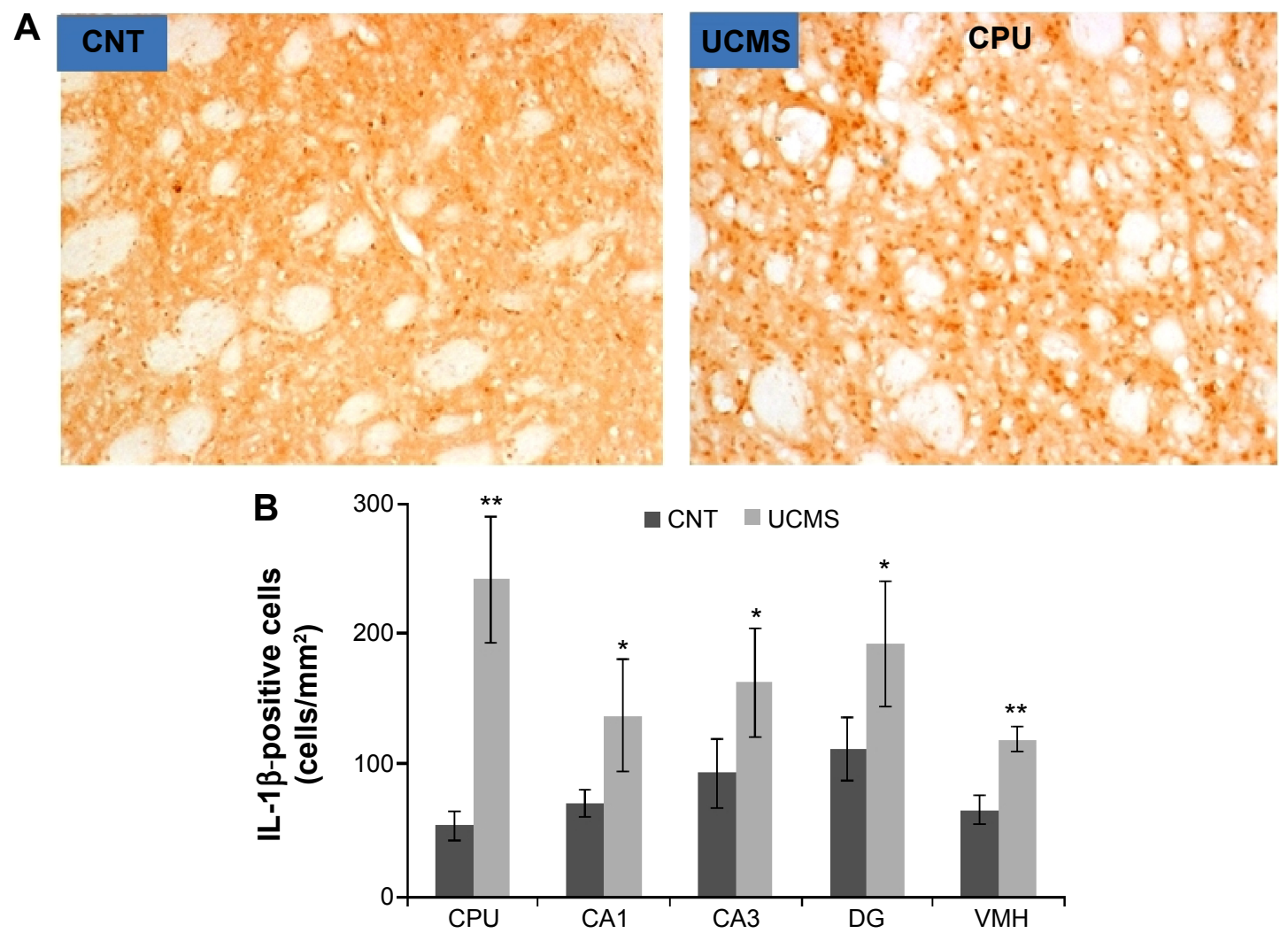

Figure 2 Increased IL-I $\beta$-positive cells in certain brain regions of stressed rats.

Notes: After the behavioral tests, the rats were sacrificed and their brains were processed for immunohistochemical staining to count the IL-I $\beta$-positive cells in the brain. The stressed rats showed more IL-I $\beta$-positive cells in the CPU, hippocampus, and VMH when compared with the CNT group. (A) Two representative photographs taken at the CPU of a control rat and a stressed (UCMS) rat. (B) Histogram of quantitative data for IL-I $\beta$-positive cells. The data are summarized as the mean \pm standard error of the mean. $* P<0.05, * * P<0.01$.

Abbreviations: CNT, control; CPU, caudate putamen; DG, dentate gyrus; IL-I $\beta$, interleukin-I beta; UCMS, unpredictable chronic mild stress; VMH, ventromedial hypothalamus. 

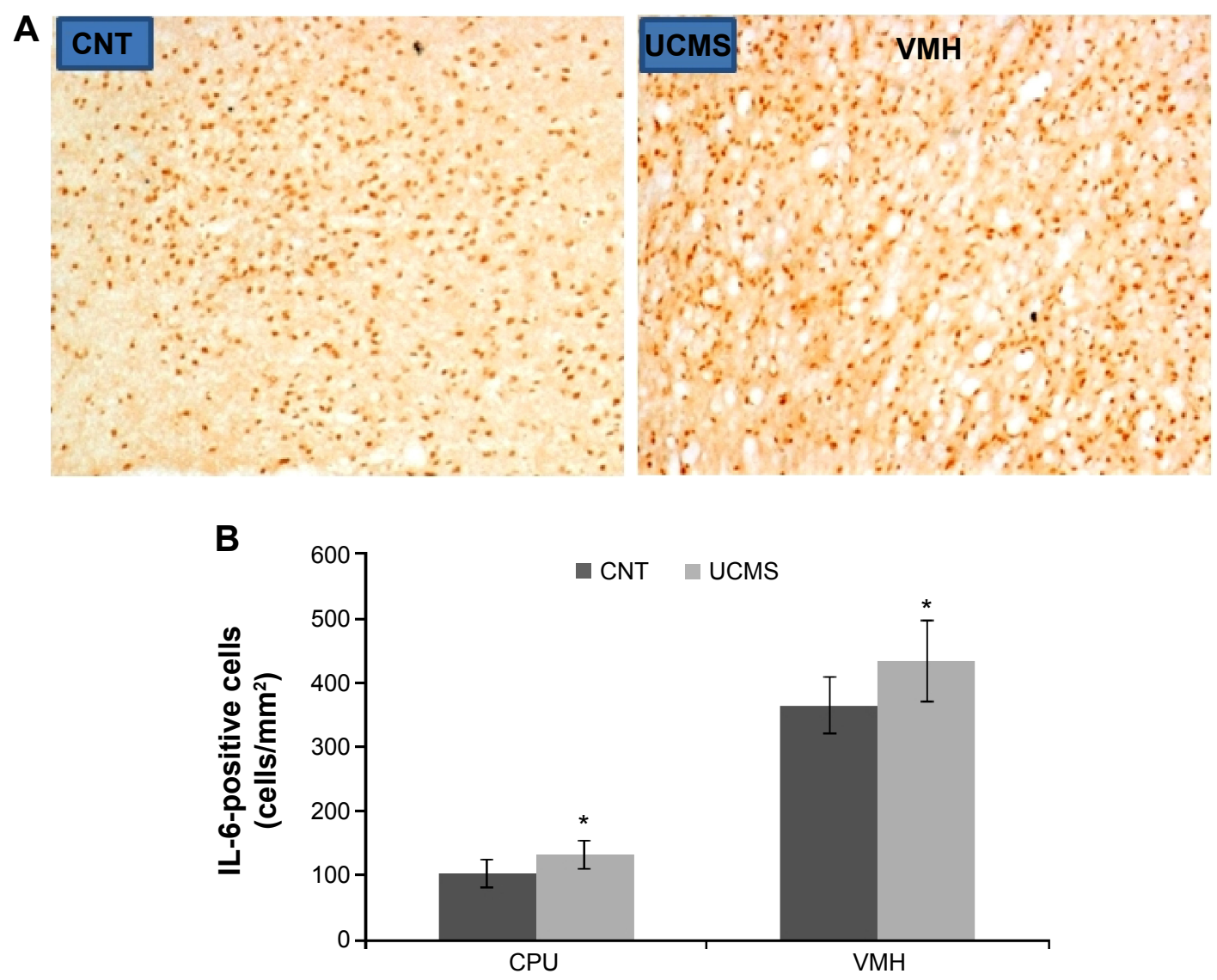

Figure 3 Increased numbers of IL-6-positive cells in certain brain regions of stressed rats.

Notes: After the behavioral tests, the rats were sacrificed and their brains were processed for immunohistochemical staining to count the IL-6-positive cells in the brain. The stressed rats showed more IL-6-positive cells in the CPU and VMH when compared with the CNT group. (A) Two representative photographs taken at the VMH of a control rat and a stressed (UCMS) rat. (B) Histogram of quantitative data for IL-6-positive cells. The data are summarized as the mean \pm standard error of the mean. $* p<0.05$.

Abbreviations: CNT, control; CPU, caudate putamen; IL, interleukin; UCMS, unpredictable chronic mild stress; VMH, ventromedial hypothalamus.

sucrose consumption and preference. In addition, the stressed rats showed higher levels of anxiety compared with controls. In line with this finding, UCMS caused anxiety-like behaviors in the elevated plus maze and open-field test in mice in another recent study. ${ }^{39}$ Together, these studies provide good face validity for the UCMS as an animal model of MDD, although the procedures of the UCMS applied in the different studies are not identical. Indeed, UCMS has been widely used to measure the antidepressant effects of various potential medications and treatments in preclinical studies. ${ }^{24-26,37-39}$

In the present study, stressed rats showed many more IL-1 $\beta$-positive cells in the CPU, hippocampus, and VMH, and a higher density of IL-6-positive cells in the CPU and $\mathrm{VMH}$, when compared with controls. These findings are in accordance with previous preclinical studies in which chronic stress increased IL-1 $\beta$ levels in several brain regions, including the hypothalamus and hippocampus. ${ }^{40,41}$ Similarly, microglial activation was seen in various brain regions of stressed mice in a recent study, including the cerebral cortex,
CPU, hippocampus, and amygdala. ${ }^{24}$ In a more recent study, mRNA expression levels of IL-1 $\beta$ and IL- 6 in the frontal cortex and hippocampus of rats were increased by UCMS. The same procedure also increased protein levels of these two cytokines in the same brain regions. ${ }^{26}$

UCMS markedly elevated levels of TNF- $\alpha$ in the PFC, $\mathrm{VMH}$, and hippocampus. This finding extended the results of a recent study showing that chronic stress led to an increase in TNF- $\alpha$ mRNA in the rat hippocampus, cortex, and hypothalamus. ${ }^{42}$ Serum TNF- $\alpha$ was also markedly increased in stressed mice as compared with controls. ${ }^{37} \mathrm{In}$ another previous study, high levels of TNF- $\alpha$ coexisted with oxidative stress in the frontal cortex of mice injected with methamphetamine. ${ }^{43}$ Together, all these studies implicate TNF- $\alpha$ in stressed conditions including UCMS.

LFB-PAS staining revealed no obvious demyelination in the brains of the stressed rats, although the number of OLs was decreased in the PFC and MBP immune reactivity was reduced in the PFC and external capsule. These results 

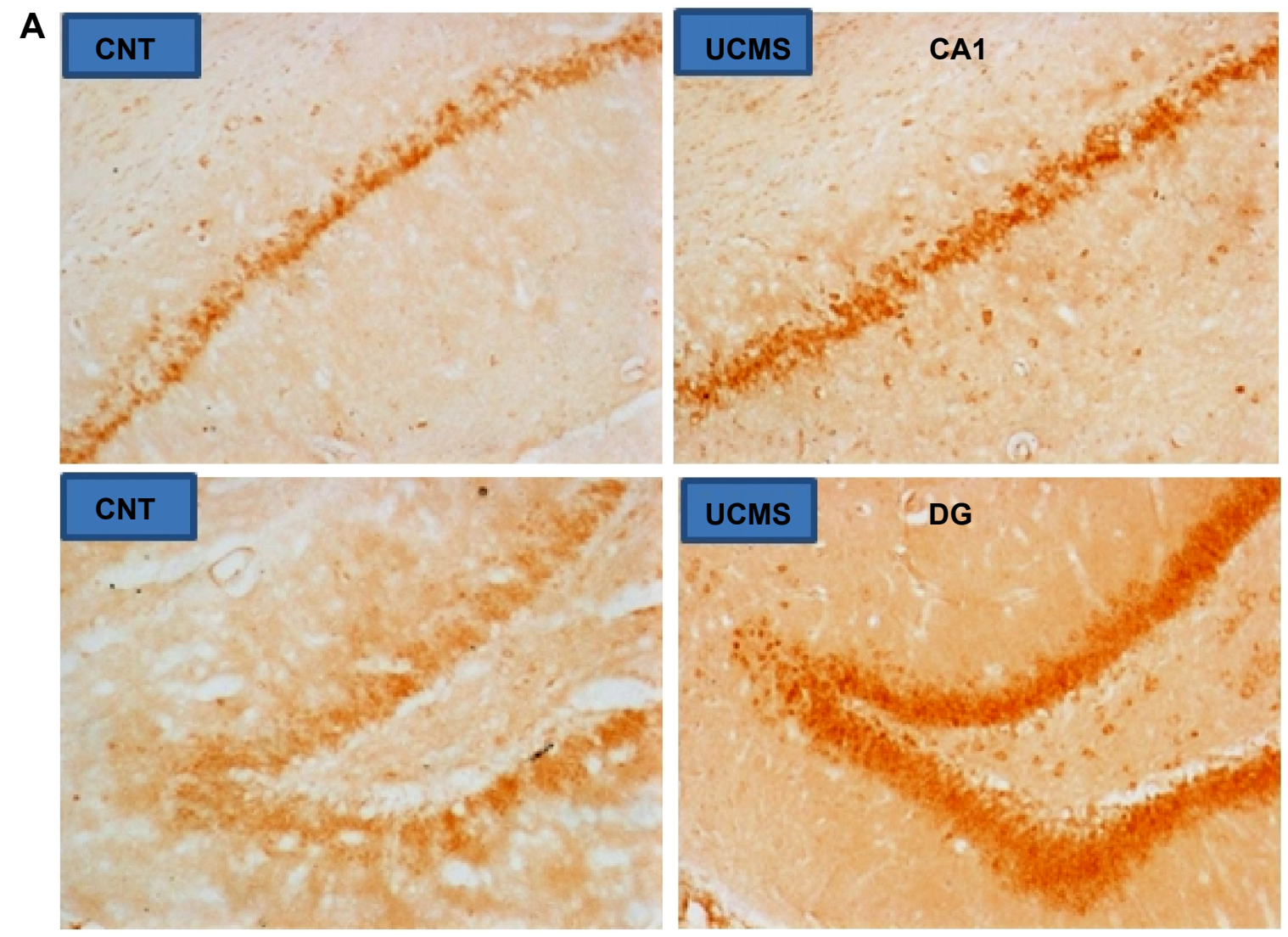

B

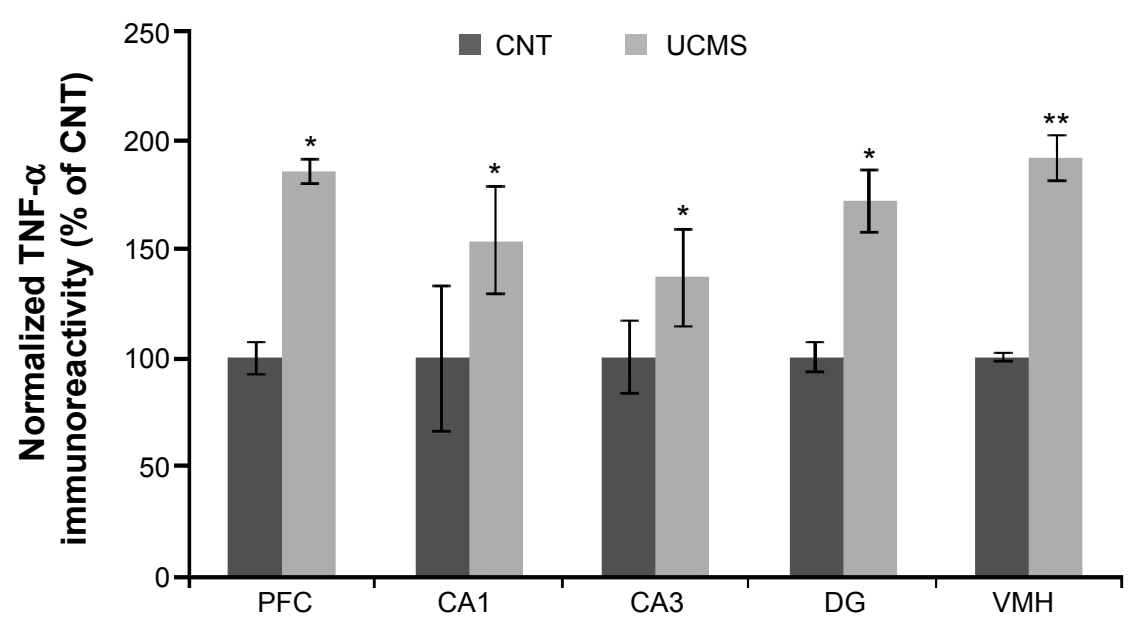

Figure 4 Increased TNF- $\alpha$ immunoreactivity in certain brain regions of stressed rats.

Notes: After the behavioral tests, the rats were sacrificed and their brains were processed for immunohistochemical staining to examine the immunoreactivity of the proinflammatory cytokine TNF- $\alpha$. The stressed rats showed higher TNF- $\alpha$ immunoreactivity in the PFC, hippocampus, and VMH when compared with the CNT group. A) Representative photographs taken at the CAI and dentate gyrus of the hippocampus of a control rat and a stressed (UCMS) rat. (B) Histogram of quantitative data for normalized TNF- $\alpha$ immunoreactivity. The data are summarized as the mean \pm standard error of the mean. $* P<0.05$, $* * P<0.01$.

Abbreviations: CNT, control; DG, dentate gyrus; PFC, prefrontal cortex; TNF- $\alpha$, tumor necrosis factor alpha; UCMS, unpredictable chronic mild stress; VMH, ventromedial hypothalamus.

suggest that UCMS did not destroy the existing myelin sheath in the brain. The decreases in OL numbers and MBP immune reactivity seen in stressed rats may be due to inhibition of OL maturation and/or a prolonged myelination process, rather than death of OLs. In support of this interpretation, previous animal studies have shown reduced proliferation of OLs and NG2-positive cells in the frontal cortex by chronic stress and human studies showed no demyelination 

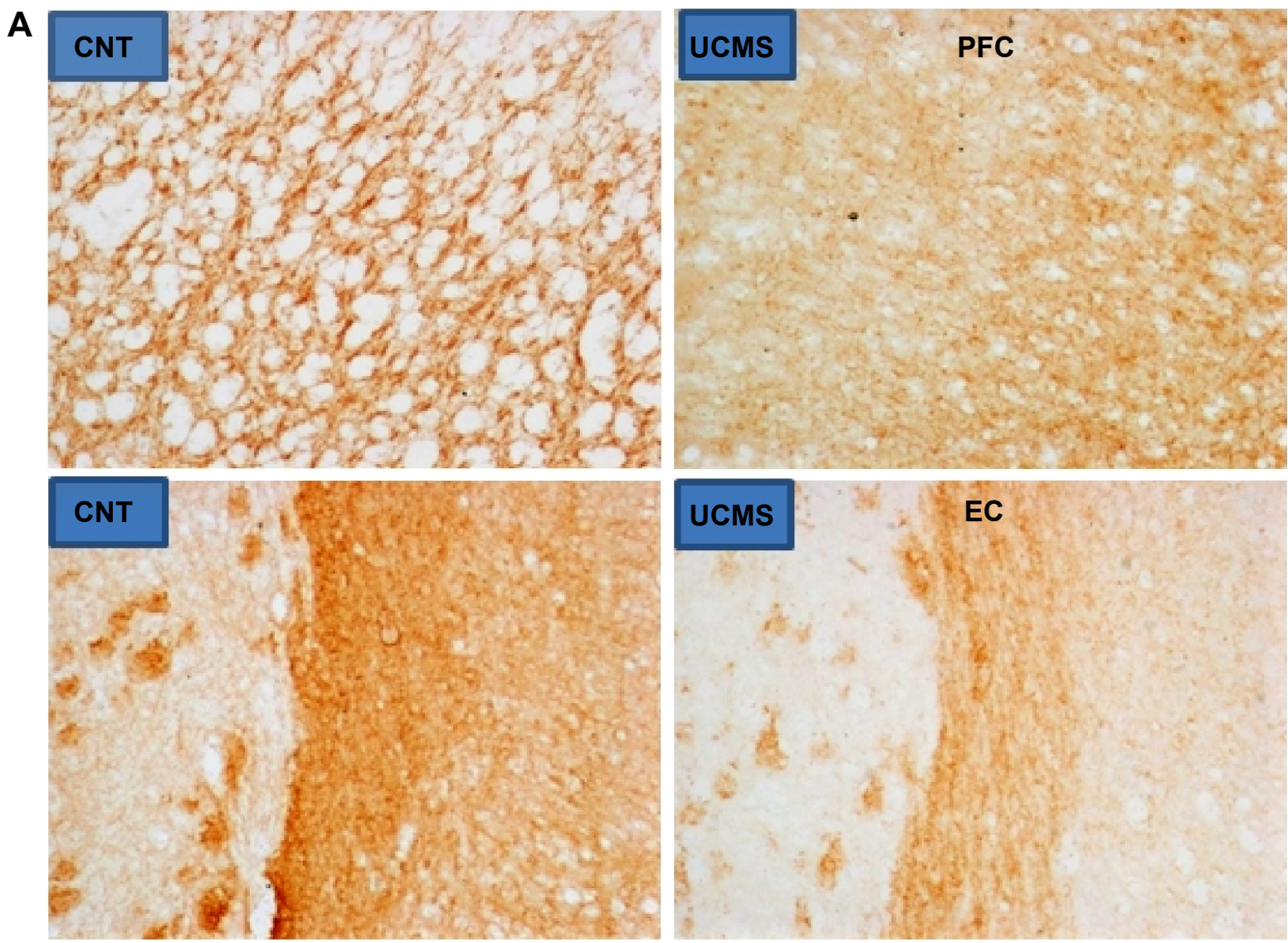

B

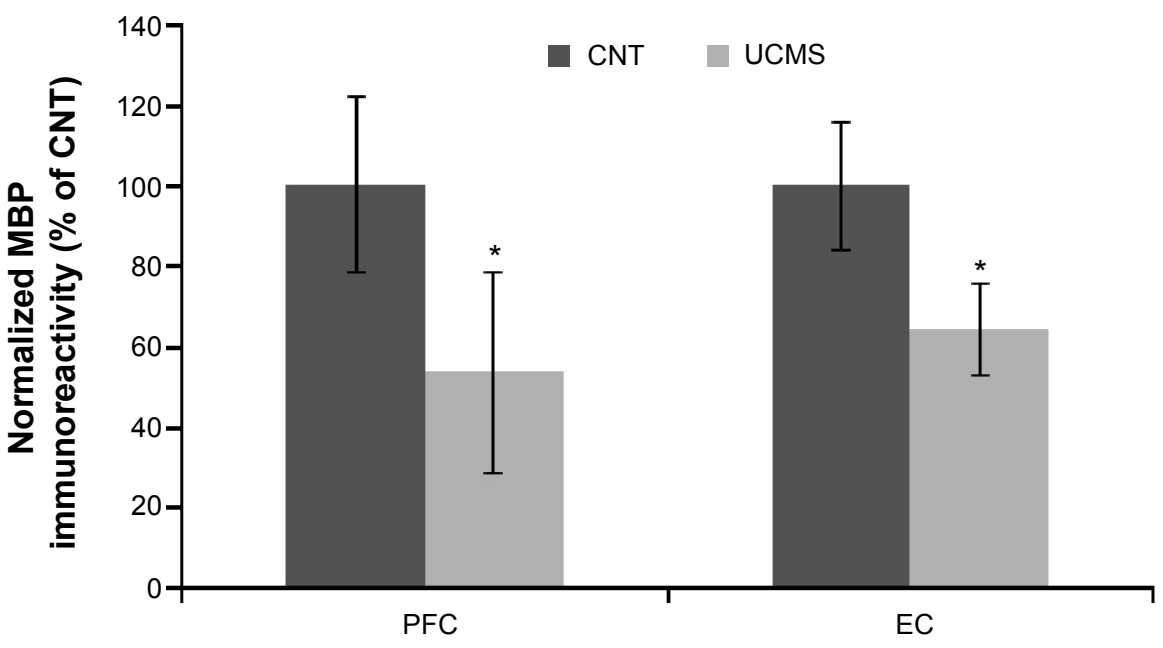

Figure 5 Decreased MBP immunoreactivity in certain brain regions of stressed rats.

Notes: After the behavioral tests, the rats were sacrificed and their brains were processed for immunohistochemical staining to examine MBP immunoreactivity. The stressed rats showed lower MBP immunoreactivity in the PFC and EC when compared with the CNT group. (A) Four representative photographs taken at the PFC and EC of a control rat and a stressed (UCMS) rat. (B) Histogram of quantitative data for normalized MBP immunoreactivity. The data are summarized as the mean \pm standard error of the mean. $* P<0.05$.

Abbreviations: CNT, control; EC, external capsule; PFC, prefrontal cortex; MBP, myelin basic protein; UCMS, unpredictable chronic mild stress.

in patients with MDD, although some studies reported reduced fractional anisotropy in the cortical regions, ${ }^{9,13,14}$ a lower density of mature OLs in the PFC,${ }^{15}$ and decreased expression of genes related to OL function in the cortex of patients with MDD. ${ }^{16}$
All the cytokines examined were significantly increased in the VMH of stressed rats. These results suggest a principal role of VMH in responding to UCMS and in regulating expression of these proinflammatory cytokines under conditions of stress. Increased cytokines, in turn, 
A

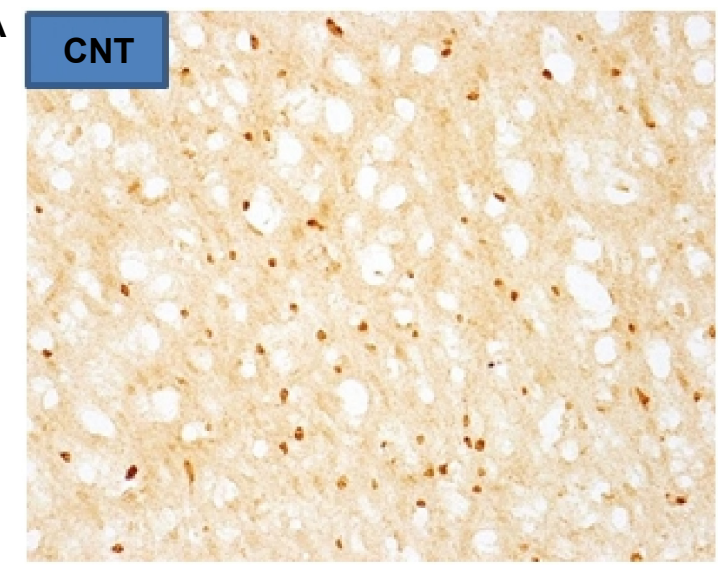

B

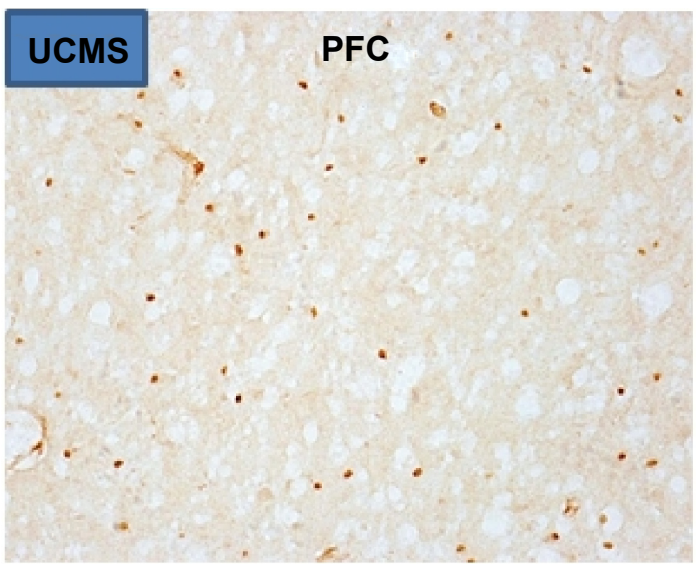

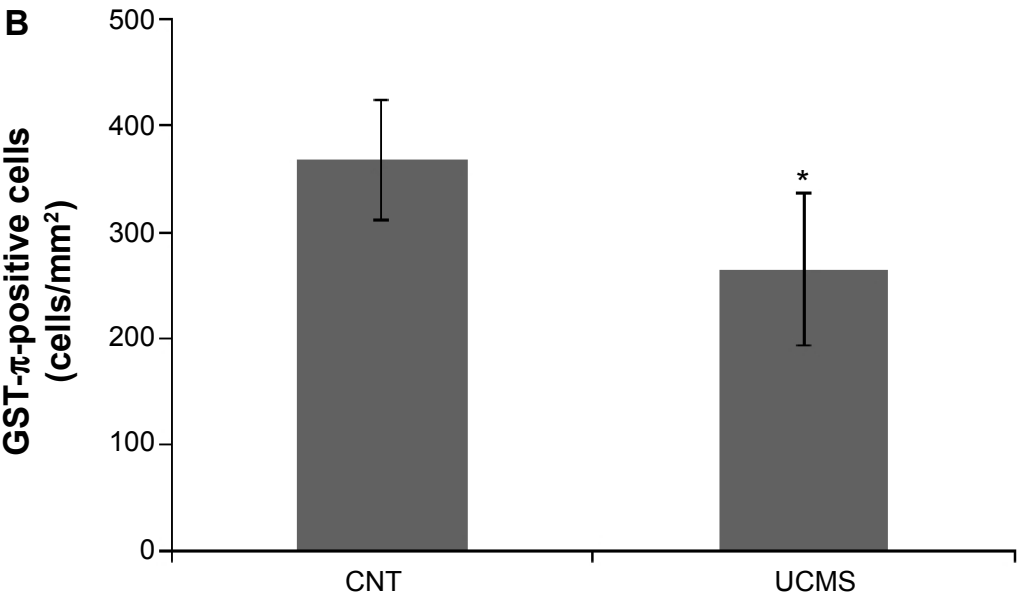

Figure 6 Decreased GST- $\pi$-positive cells in the PFC of stressed rats.

Notes: After the behavioral tests, the rats were sacrificed and their brains were processed for immunohistochemical staining to count GST- $\pi$ positive cells. The stressed rats showed fewer GST- $\pi$-positive cells in PFC, when compared with the CNT group. (A) Two representative photographs taken at the PFC of a control rat and a stressed (UCMS) rat. (B) Histogram of GST- $\pi$-positive cells. The data are summarized as the mean \pm standard error of the mean. $* P<0.05$.

Abbreviations: CNT, control; GST- $\pi$, glutathione-S-transferase- $\pi$; PFC, prefrontal cortex; UCMS, unpredictable chronic mild stress.

may mediate the functioning of the hypothalamic-pituitaryadrenal axis. For example, IL-1 $\beta$ influenced the excitability of hypothalamic parvocellular neurons in whole-cell patch clamp recordings in the rat. ${ }^{44}$ Interestingly, UCMS increased IL-1 $\beta$-positive cells in both the CPU and hippocampus, but increased IL-6-positive cells in the CPU and increased TNF- $\alpha$ immune reactivity only in the hippocampus. These different spatial profiles for the three proinflammatory cytokines investigated in stressed rats suggest that the increase in IL- $1 \beta$ might precede that of IL- 6 and TNF- $\alpha$, implying a causal relationship between the changes in IL-1 $\beta$ and the latter two cytokines. This speculation is in line with the notion that IL-1 represents an important central component operating in a neurochemical and immune network for efficient coping to prevent stress-associated psychopathology and neuropathology. ${ }^{45}$

It should be noted that UCMS-induced white matter abnormalities were seen only in the external capsule and PFC, where the immunoreactivity of TNF- $\alpha$, but not the other two cytokines, was higher. This observation indicates no active damage in these two brain regions and suggests an important role of TNF- $\alpha$ in the pathogenesis of the observed abnormal white matter in stressed rats. In support of this suggestion, an in vitro study showed that TNF- $\alpha$ was an essential factor for cuprizone to be able to decrease the viability of cultured oligodendroglial cells and that TNF- $\alpha$-treated cells showed an enhancement in oxidant production, implying a relationship between oxidative stress and increased TNF- $\alpha .{ }^{46}$ Indeed, oxidative stress coexisted with high levels of TNF- $\alpha$ in the frontal cortex of mice injected with methamphetamine. ${ }^{43}$ In addition, this psychostimulant decreased MBP immune reactivity and the number of mature OLs in the mouse brain. ${ }^{47}$ While providing evidence supporting an important role of TNF- $\alpha$ in the pathophysiology of oligodendroglial cells, these previous studies also allowed us to propose that UCMS may delay the maturation of OLs and prolong the myelination process via induction of oxidative stress. Further evidence supporting this interpretation includes the following 
data from our group and others: UCMS was shown to induce oxidative stress, evidenced by increased levels of thiobarbituric acid-reactive substance and decreased glutathione levels and enzymatic activity of superoxide dismutase and catalase in the brain; ${ }^{37,38}$ oxidative stress disrupted OL differentiation by decreasing the expression of key genes that promote OL differentiation from neural stem cells and increases the expression of genes known to inhibit differentiation; ${ }^{48}$ the myelination process was delayed by oxidative stress as a result of exposure to cuprizone, ${ }^{49}$ which inhibited mitochondrial function and caused oxidative stress in brain cells. ${ }^{50}$

Some limitations of this study should not be ignored. First, the negative results for LFB-PAS staining cannot completely rule out the presence of an abnormal myelin sheath in the stressed rats. The safest method would be electron microscopy, under which both mild damage to and minor alterations in the myelin sheath can be revealed. Second, the cellular sources of the cytokines were not definite in the present study; to determine this, the double immunofluorescence method should be employed. Third, future studies should provide direct evidence of delayed maturation of OLs and a prolonged myelination process in UCMS rats.

In summary, UCMS induced depression-like behaviors in rats and increased levels of proinflammatory cytokines (IL-1 $\beta$, IL-6, and TNF- $\alpha$ ) in various brain regions. This procedure induced white matter abnormalities in the external capsule and PFC in the rat brain, but caused no demyelination. The uncoupled white matter abnormality and changes in IL-1 $\beta$ and IL- 6 in the brain regions suggest that there is no causal relationship between the two events under conditions of stress. The concurrent white matter abnormality and TNF- $\alpha$ increase in the PFC suggests that TNF- $\alpha$ may be an important factor in the pathogenesis of the observed white matter abnormality in stressed rats. The results of this study, our own previous data, and those of other groups suggest it is likely that UCMS, via oxidative stress, delays the maturation of OLs and/or prolongs the myelination process in the brain.

\section{Acknowledgment}

This study was supported by a grant from the National Natural Science Foundation of China (30770771).

\section{Disclosure}

The authors report no conflicts of interests in this work.

\section{References}

1. Wetzler S, Kahn R, Strauman TJ, Dubro A. Diagnosis of major depression by self-report. J Pers Assess. 1989;53:22-30.

2. Schechter LE. Major depressive disorder. Curr Pharm Des. 2005;11: 143-144.
3. Dantzer R, Wollman E, Vitkovic L, Yirmiya R. Cytokines and depression: fortuitous or causative association. Mol Psychiatry. 1999;4: 328-332.

4. Serafini G, Pompili M, Elena Seretti M, et al. The role of inflammatory cytokines in suicidal behavior: a systematic review. Eur Neuropsychopharmacol. 2013;23:1672-1686.

5. Miller AH, Maletic V, Raison CL. Inflammation and its discontents: the role of cytokines in the pathophysiology of major depression. Biol Psychiatry. 2009;65:732-741.

6. Miller AH. Depression and immunity: a role for T cells? Brain Behav. Immun. 2010;24:1-8.

7. Piser TM. Linking the cytokine and neurocircuitry hypotheses of depression: a translational framework for discovery and development of novel anti-depressants. Brain Behav Immun. 2010;4:515-524.

8. Tyring S, Gottlieb A, Papp K, et al. Etanercept and clinical outcomes, fatigue, and depression in psoriasis: double-blind placebo controlled randomised phase III trial. Lancet. 2006;367:29-35.

9. Colin A, Reggers J, Castronovo V, Ansseau M. [Lipids, depression and suicide]. Encephale. 2003;29:49-58. French.

10. Li L, Ma N, Li Z, et al. Prefrontal white matter abnormalities in young adult with major depressive disorder: a diffusion tensor imaging study. Brain Res. 2007;1168:124-128.

11. Ma N, Li L, Shu N, et al. White matter abnormalities in first-episode, treatment-naive young adults with major depressive disorder. $\mathrm{Am} \mathrm{J}$ Psychiatry. 2007;164:823-826.

12. Tham MW, Woon PS, Sum MY, Lee TS, Sim K. White matter abnormalities in major depression: evidence from post-mortem, neuroimaging and genetic studies. J Affect Disord. 2011;132:26-36.

13. Zou K, Huang X, Li T, et al. Alterations of white matter integrity in adults with major depressive disorder: a magnetic resonance imaging study. J Psychiatry Neurosci. 2008;33:525-530.

14. Kiesepp T, Eerola M, Mäntylä R, et al. Major depressive disorder and white matter abnormalities: a diffusion tensor imaging study with tractbased spatial statistics. J Affect Disord. 2010;120:240-244.

15. Uranova NA, Vostrikov VM, Orlovskaya DD, Rachmanova VI. Oligodendroglial density in the prefrontal cortex in schizophrenia and mood disorders: a study from the Stanley Neuropathology Consortium. Schizophr Res. 2004;67:269-275.

16. Aston C, Jiang L, Sokolov BP. Transcriptional profiling reveals evidence for signaling and oligodendroglial abnormalities in the temporal cortex from patients with major depressive disorder. Mol Psychiatry. 2005; 10:309-322.

17. Savignac HM, Hyland NP, Dinan TG, Cryan JF. The effects of repeated social interaction stress on behavioural and physiological parameters in a stress-sensitive mouse strain. Behav Brain Res. 2011;216: 576-584.

18. Fu X, Zunich SM, O’Connor JC, Kavelaars A, Dantzer R, Kelley KW. Central administration of lipopolysaccharide induces depressive-like behavior in vivo and activates brain indoleamine 2, 3 dioxygenase in murine organotypic hippocampal slice cultures. J Neuroinflammation. 2010;7:43.

19. Rizzo A, Bevilacqua N, Guida L, et al. Effect of resveratrol and modulation of cytokine production on human periodontal ligament cells. Cytokine. 2012;60:197-204.

20. Banasr M, Valentine GW, Li XY, Gourley SL, Taylor JR, Duman RS. Chronic unpredictable stress decreases cell proliferation in the cerebral cortex of the adult rat. Biol Psychiatry. 2007;62:496-504.

21. Czeh B, Muller-Keuker JI, Rygula R, et al. Chronic social stress inhibits cell proliferation in the adult medial prefrontal cortex: hemispheric asymmetry and reversal by fluoxetine treatment. Neuropsychopharmacology. 2007;32:1490-1503.

22. Alonso G. Prolonged corticosterone treatment of adult rats inhibits the proliferation of oligodendrocyte progenitors present throughout white and gray matter regions of the brain. Glia. 2000;31:219-231.

23. Wennström M, Hellsten J, Ekstrand J, Lindgren H, Tingström A. Corticosterone-induced inhibition of gliogenesis in rat hippocampus is counteracted by electroconvulsive seizures. Biol Psychiatry. 2006;59: $178-186$. 
24. Farooq RK, Isingrini E, Tanti A, et al. Is unpredictable chronic mild stress (UCMS) a reliable model to study depression-induced neuroinflammation? Behav Brain Res. 2012;231:130-137.

25. Liu W, Sheng H, Xu Y, et al. Swimming exercise ameliorates depressionlike behavior in chronically stressed rats: relevant to pro-inflammatory cytokines and IDO activation. Behav Brain Res. 2013;242:110-116.

26. Liu XL, Luo L, Liu BB, et al. Ethanol extracts from Hemerocallis citrina attenuate the upregulation of pro-inflammatory cytokines and indoleamine 2,3-dioxygenase in rats. J Ethnopharmacol. 2014;153:484-490.

27. Mutlu O, Gumuslu E, Ulak G, et al. Effects of fluoxetine, tianeptine and olanzapine on unpredictable chronic mild stress-induced depression-like behavior in mice. Life Sci. 2012;91:1252-1262.

28. Bekris S, Antoniou K, Daskas S, Papadopoulou-Daifoti Z. Behavioural and neurochemical effects induced by chronic mild stress applied to two different rat strains. Behav Brain Res. 2005;161:45-59.

29. Paxinos G, Watson C. The Rat Brain in Stereotaxic Coordinates. 3rd ed. New York, NY, USA: Elsevier Academic Press; 1997.

30. Zhang Y, Xu H, Jiang W, et al. Quetiapine alleviates the cuprizoneinduced white matter pathology in the brain of C57BL/6 mouse. Schizophr Res. 2008;106:182-191.

31. Xu H, Yang HJ, Zhang Y, Clough R, Browning R, Li XM. Behavioral and neurobiological changes in C57BL/6 mice exposed to cuprizone. Behav Neurosci. 2009;123:418-429.

32. Yang HJ, Wang H, Zhang Y, et al. Region-specific susceptibilities to cuprizone-induced lesions in the mouse forebrain: implications for the pathophysiology of schizophrenia. Brain Res. 2009;1270:121-130.

33. Xu H, Qing H, Lu W, et al. Quetiapine attenuates the immobilization stress-induced decrease of brain-derived neurotrophic factor expression in rat hippocampus. Neurosci Lett. 2002;321:65-68.

34. Mizoguchi K, Ishige A, Aburada M, Tabira T. Chronic stress attenuates glucocorticoid negative feedback: involvement of the prefrontal cortex and hippocampus. Neuroscience. 2003;119:887-897.

35. Elbejjani M, Fuhrer R, Abrahamowicz M, et al. Hippocampal atrophy and subsequent depressive symptoms in older men and women: results from a 10-year prospective cohort. Am J Epidemiol. 2014; 180:385-393.

36. Tanebe K, Nishijo H, Muraguchi A, Ono T. Effects of chronic stress on hypothalamic interleukin-1beta, interleukin-2, and gonadotrophinreleasing hormone gene expression in ovariectomized rats. J Neuroendocrinol. 2000;12:13-21.

37. Kumar B, Kuhad A, Chopra K. Neuropsychopharmacological effect of sesamol in unpredictable chronic mild stress model of depression: behavioral and biochemical evidences. Psychopharmacology (Berl). 2011;214: $819-828$
38. Schaalan MF, Nassar NN. Effects of octreotide in chronically mild stressed rats: possible role of immune and oxidative stress pathways. Neurochem Res. 2011;36:1717-1723.

39. Jindal A, Mahesh R, Bhatt S. Etazolate rescues behavioral deficits in chronic unpredictable mild stress model: modulation of hypothalamicpituitary-adrenal axis activity and brain-derived neurotrophic factor level. Neurochem Int. 2013;63:465-475.

40. Nguyen KT, Deak T, Owens SM, et al. Exposure to acute stress induces brain interleukin-1beta protein in the rat. J Neurosci. 1998;18:2239-2246.

41. Grippo AJ, Francis J, Beltz TG, Felder RB, Johnson AK. Neuroendocrine and cytokine profile of chronic mild stress-induced anhedonia. Physiol Behav. 2005;84:697-706.

42. You Z, Luo C, Zhang W, et al. Pro- and anti-inflammatory cytokines expression in rat's brain and spleen exposed to chronic mild stress: involvement in depression. Behav Brain Res. 2011;225:135-141.

43. Flora G, Lee YW, Nath A, Maragos W, Hennig B, Toborek M. Methamphetamine-induced TNF-alpha gene expression and activation of AP-1 in discrete regions of mouse brain: potential role of reactive oxygen intermediates and lipid peroxidation. Neuromolecular Med. 2002;2: 71-85.

44. Ferri CC, Ferguson AV. Interleukin-1 beta depolarizes paraventricular nucleus parvocellular neurones. J Neuroendocrinol. 2003;15:126-133.

45. Gdek-Michalska A, Bugajski J. Interleukin-1 (IL-1) in stress-induced activation of limbic-hypothalamic-pituitary adrenal axis. Pharmacol Rep. 2010;62:969-982.

46. Pasquini LA, Calatayud CA, Bertone U, et al. The neurotoxic effect of cuprizone on oligodendrocytes depends on the presence of proinflammatory cytokines secreted by microglia. Neurochem Res. 2007;32: 279-292.

47. Yang HJ, Wang L, Cheng Q, Xu H. Abnormal behaviors and microstructural changes in white matter of juvenile mice repeatedly exposed to amphetamine. Schizophr Res Treatment. 2011;2011:542896.

48. French HM, Reid M, Mamontov P, Simmons RA, Grinspan JB. Oxidative stress disrupts oligodendrocyte maturation. J Neurosci Res. 2009;87:3076-3087.

49. Xu H, Yang HJ, Li XM. Differential effects of antipsychotics on the development of rat oligodendrocyte precursor cells exposed to cuprizone. Eur Arch Psychiatry Clin Neurosci. 2014;264:121-129.

50. Xuan Y, Yan G, Peng H, Wu R, Xu H. Concurrent changes in (1)H MRS metabolites and antioxidant enzymes in the brain of C57BL/6 mouse short-termly exposed to cuprizone: possible implications for schizophrenia. Neurochem Int. 2014;69:20-27.
Neuropsychiatric Disease and Treatment

\section{Publish your work in this journal}

Neuropsychiatric Disease and Treatment is an international, peerreviewed journal of clinical therapeutics and pharmacology focusing on concise rapid reporting of clinical or pre-clinical studies on a range of neuropsychiatric and neurological disorders. This journa is indexed on PubMed Central, the 'PsycINFO' database and CAS,

\section{Dovepress}

and is the official journal of The International Neuropsychiatric Association (INA). The manuscript management system is completely online and includes a very quick and fair peer-review system, which is all easy to use. Visit http://www.dovepress.com/testimonials.php to read real quotes from published authors. 